\title{
Impacts of Spike Shape Variations on Synaptic Communication
}

\author{
Hamideh Ramezani, Student Member, IEEE and Ozgur B. Akan, Fellow, IEEE
}

\begin{abstract}
Understanding the communication theoretical capabilities of information transmission among neurons, known as neuro-spike communication, is a significant step in developing bio-inspired solutions for nanonetworking. In this study, we focus on a part of this communication known as synaptic transmission for pyramidal neurons in Cornu Ammonis (CA) area of hippocampus location in the brain and propose a communication-based model for it that includes effects of spike shape variation on neural calcium signaling and the vesicle release process downstream of it. For this aim, we find impacts of spike shape variation on opening of voltage-dependent calcium channels (VDCCs), which control the release of vesicles from pre-synaptic neuron by changing the influx of calcium ions. Moreover, we derive the structure of optimum receiver based on Neyman-Pearson detection method to find the effects of spike shape variations on the functionality of neuro-spike communication. Numerical results depict that changes in both spike width and amplitude affect the error detection probability. Moreover, these two factors do not control the performance of the system independently. Hence, a proper model for neuro-spike communication should contain effects of spike shape variations during axonal transmission on both synaptic propagation and spike generation mechanisms to enable us to accurately explain the performance of this communication paradigm.
\end{abstract}

Index Terms-Nanoscale neuro-spike communication, Spike shape variation, Vesicle release process, Synaptic channel

\section{INTRODUCTION}

The nervous system is a huge communication network of nerve cells, i.e., neurons, which controls all of the actions of the body effectively. Hence, understanding and analyzing communication and network theoretical capabilities and shortcomings of this network help us to find a bio-stable and biocompatible nanoscale communication paradigm [1]. Neurons act as transceivers in this system and can be considered as nanomachines. Hence, the first step toward reaching this paradigm is understanding the basics of communication among neurons, i.e., nanoscale neuro-spike communication [2].

Neuro-spike communication contains three phases: axonal propagation, synaptic transmission, and spike generation [2]. As depicted in Fig. 1, three main parts of each neuron

H. Ramezani is with the Next-generation and Wireless Communications Laboratory (NWCL), Department of Electrical and Electronics Engineering, Koç University, Istanbul 34450, Turkey (e-mail: hramezani13@ku.edu.tr).

O. B. Akan is with the Internet of Everything (IoE) Group, Electrical Engineering Division, Department of Engineering, University of Cambridge, Cambridge CB3 0FA, U.K. and also with NWCL, Department of Electrical and Electronics Engineering, Koç University, Istanbul 34450, Turkey (e-mail: oba21@cam.ac.uk).

This work was supported in part by ERC Project MINERVA under grant ERC-2013-CoG 616922, by the EU Project CIRCLE under grant EU-H2020FET-Open 665564 and by TÜBITAK graduate scholarship program under grant BIDEB-2215.

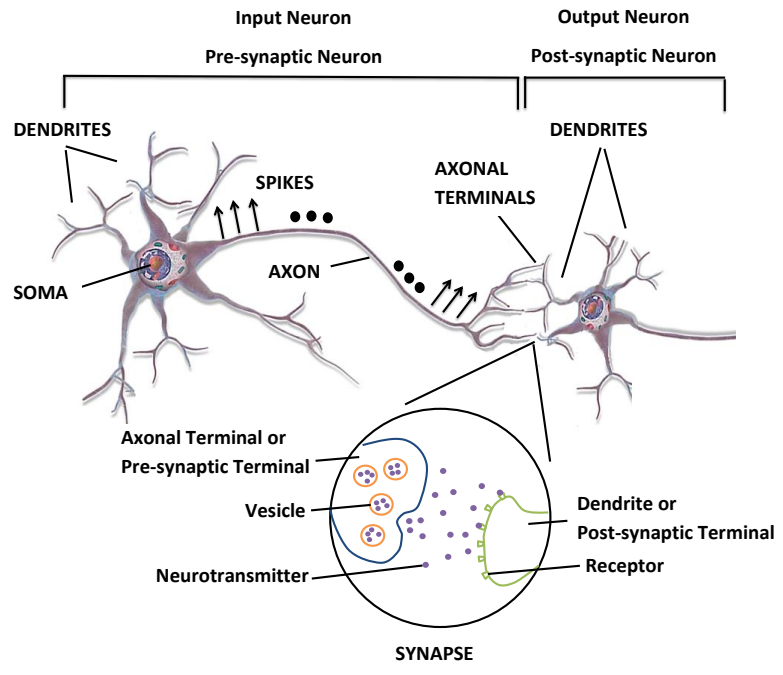

Fig. 1. Nueral anatomy of communication among a pair of neurons.

participate in this communication: dendrite, soma, and axon. Neurons receive stimulation from their dendrites. In the spike generation phase, if the received stimulation is strong enough, the neuron fires a molecular impulse signal known as action potential (AP) or spike. The membrane potential changes during an AP are shown in Fig. 2. The AP, which is information carrier in neuro-spike communication, passes through the axon until reaching the axonal terminals in the axonal propagation phase [3]. While the shape of spike is independent from the strength of the applied stimulus, depending on the memory of the axon from previously propagated spikes, both peak amplitude and width of spike can change during axonal transmission [4]-[6]. Arrival of a spike to the axonal terminals moves the neuron to the synaptic transmission phase, which is the focus of this study. In this phase, voltage-dependent calcium channels (VDCCs) located in the axonal terminal open as a result of spike arrival and allow influx of calcium ions to the pre-synaptic terminal. This initiates the release of vesicles, groups of chemical substances known as neurotransmitters, to the synapse, i.e., the gap between axonal terminals and the receiver neuron [3]. Variations in the shape of the pre-synaptic spike modulate the concentration of calcium ions, $\mathrm{Ca}^{2+}$, in the terminal, which, in turn, affects neurotransmitter release to the synapse [7]. The released neurotransmitters diffuse through synapse, some of them stimulate the receiver neuron by binding to its receptors, others leave the synapse.

Few studies exist in the literature on modeling and understanding synaptic transmission based on engineering per- 


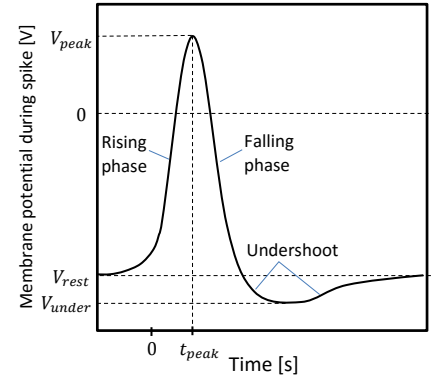

Fig. 2. Membrane potential changes during an action potential [3].

spective. In [8], a transfer function is derived for synaptic transmission by considering 1-dimensional diffusion of neurotransmitters in synapse and modeling the binding of receptors. Later on, the same authors analyzed the impact of the noise on synaptic transmission in [9]. In a more in depth study, the effect of the pre-synaptic calcium concentration on the release probability is analyzed in [10]. However, the possibility of the change in the AP shape is not considered in this paper and the changes in the calcium concentration is proposed to be a controlling option for vesicle release process. Moreover, the relation of release probability and calcium influx is missing in this study. In [2], the synaptic transmission is modeled and analyzed by defining finite number of states for vesicle release process and utilizing 1-dimensional model for neurotransmitters diffusion in synapse. Moreover, the multipleaccess to a post synaptic neuron through multiple synapses is investigated in [11], where the dependence of the rate region of synaptic communication channel on dynamics of neuronal spiking and vesicle release process is studied. However, the impact of spike shape on neuronal calcium signaling and its consequent effect on release process has not been considered in the aforementioned studies. In our previous work [7], we investigated the impact of changes in the spike shape on vesicle release probability. Moreover, in [12], we proposed the first synaptic channel model that investigates outcomes of AP width variation on the performance of this communication channel. In this work, we expand the model to accommodate the effects of variation in both amplitude and width of spike. Since changes in spike shape affect the percentage of opened VDCCs and their opening time [13] we model the gating of VDCCs and its impact on the release of neurotransmitters in this study. Moreover, we find the probability of error detection by assuming that the receiver neuron detects spikes based on Neyman-Pearson method. The error detection probability is then used to find the effect of spike shape variation on the efficiency of the neuro-spike communication. Simulation results depict that spike width and amplitude variations have a coupled impact on the performance of the neuro-spike communication. Hence, a proper model for neuro-spike communication should contain effects of spike shape variations during axonal transmission on both synaptic propagation and spike generation mechanisms.

The remainder of the paper is as follows. The fundamental biological processes involved in synaptic communication are presented in Section II. Then, the synaptic communication model is proposed and the structure of optimum detector is derived in Section III. The effect of spike shape on functionality of the proposed model is evaluated in Section IV. Finally, the paper is concluded in Section V.

\section{BIOLOGICAL BACKGROUND}

Synaptic transmission contains three main parts, vesicle release, neurotransmitter diffusion, and post-synaptic neuron stimulation. In this section, we present the biological events of each of these processes to provide the fundamental basis needed to propose a realistic model for synaptic transmission.

a) Vesicle Release: Arrival of the spike to the axonal terminal changes its membrane potential. When the membrane potential changes are strong enough, the VDCCs open and allow the entering of the calcium ions to the pre-synaptic terminal. The resulting elevation in the concentration of internal $\mathrm{Ca}^{2+}$ near the synaptic vesicles that are ready to be released initiates the fusion of these vesicles to the pre-synaptic membrane. This results in the release of the vesicle contents, i.e., neurotransmitters, to the synaptic cleft [3]. Various type of VDCCs having different channel characteristics exist in each neuron. According to [14], P/Q-, R-, and N-type VDCCs are responsible for entrance of $\mathrm{Ca}^{2+}$ to the axonal terminal of hippocampal pyramidal neurons upon arrival of an AP.

Not all of the existing vesicles in an axonal terminal are ready to be release upon arrival of the spike. Based on [15], the vesicles, containing a fixed amount of neurotransmitters docked in them [16], are grouped to at least two distinct pools, (i) a pool of readily releasable vesicles (RRVs) called ready pool (RP), which contains only a small percentage of all vesicles, and (ii) a larger pool containing the vesicles farther from the pre-synaptic site. Each AP can initiate the release of at most one RRV from each release terminal in the hippocampal pyramidal neurons [17], which is refilled by the vesicles nearer to the release site [18], [19] after each release.

b) Neurotransmitter Diffusion: By release of vesicles, the electrical signal that neuron received from environment is changed to the chemical signal. Moreover, the information is transmitted to the post-synaptic neuron through diffusion of neurotransmitters in synaptic cleft. As a result of the randomness in the diffusion process, number of the neurotransmitters that reach post-synaptic neuron changes from trial to trial.

c) Post-Synaptic Neuron Stimulation: The neurotransmitters that reach the post-synaptic neuron bind to receptors of this neuron and open ionic channels allowing the flow of ions, which changes the membrane potential of the neuron, i.e., stimulates it. The maximum amount of this membrane potential change varies from trial to trial as a result of the randomness in the diffusion process, number of available receptors, and ionic flows. Moreover, this membrane potential change can be positive or negative, happening in excitatory and inhibitory synapses, respectively. Excitatory synapses are the most abundant type in the hippocampus [20]. Furthermore, among the excitatory stimulation sources in hippocampal pyramidal neurons, the response of AMPA and NMDA receptors are dominant [3], and the ratio of AMPA and NMDA receptors current within different synapses to a neuron is constant [21]. 


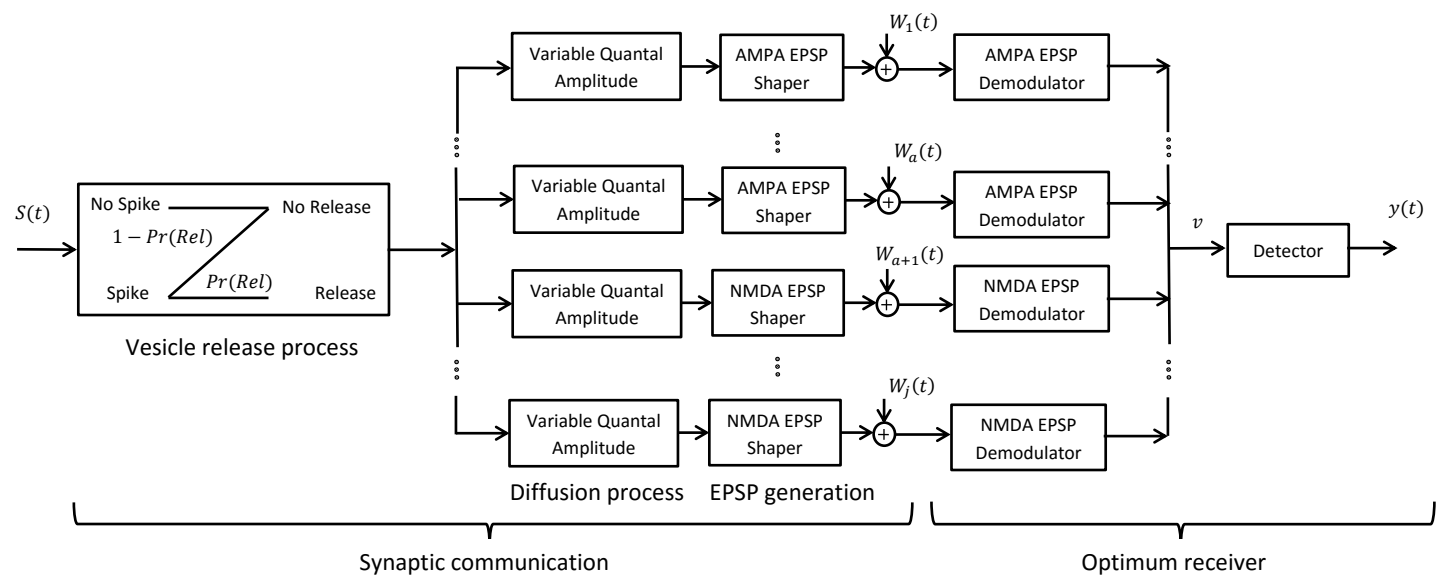

Fig. 3. Synaptic propagation with optimum receiver for spike generation.

The received stimulations in dendrites propagate through it until reaching the soma. Although the stimulation degrades during propagation along dendrites, the amplitude of the excitatory stimulation after reaching the soma does not depend on the location of synapse in hippocampal pyramidal neurons due to different biological mechanisms [22]-[24].

Before stimulation, the membrane potential of the soma is at rest, i.e, $V_{\text {rest }}$. If the received stimulation exceeds a threshold, the neuron fires a spike [3]. The spike is then propagated thorough axon to be transmitted to other neurons by axonal terminals. A neuron, which is not stimulated during last seconds, is in control situation and the shape of propagating spikes through its axon is roughly constant. However, the spike shape vary during axonal propagation as a result of the memory of neuron from previous stimulations [4].

\section{Model Description}

The overall channel model of synaptic transmission is depicted in Fig. 3, where $S(t)$, which contains spikes with different shapes, is the input to pre-synaptic terminals. Here, we first explain the Hodgkin-Huxley $(\mathrm{H}-\mathrm{H})$ model, which is used to derive the spike shape in control situation. Then, we describe the channel model of each of the processes involved in synaptic communication. Finally, we derive the structure of the optimum receiver for our model.

\section{A. Spike Shape in Control Situation}

To derive the spike shape in control situation, we utilize the Hodgkin-Huxley ionic current equations, which show how the membrane responds to the stimuli [25]. Since the shape of the spike can change during axonal propagation [4], we utilize the derived spike shape from $\mathrm{H}-\mathrm{H}$ model as the spike shape in control situation and find the impact of changes in the amplitude and duration of this shape on the performance of the synaptic communication.

In $\mathrm{H}-\mathrm{H}$ model, the membrane current, i.e., $I_{m}(t)$, is derived by considering two components, (i) ionic current as a result of Potassium, Sodium, and leakage ions, which are noted as $I_{K}(t), I_{N a}(t)$, and $I_{L}(t)$, respectively, and (ii) the current associated with charging or discharging the membrane capacitance, i.e., $I_{C}(t)$. Hence, $I_{m}(t)$ can be written as follows [25].

$$
I_{m}(t)=I_{K}(t)+I_{N a}(t)+I_{L}(t)+I_{C}(t) .
$$

The capacitance current can be written as $I_{C}(t)=C_{m} \frac{d V_{m}(t)}{d t}$, where $C_{m}$ is the membrane capacitance and $V_{m}(t)$ is the membrane potential at time $t$. Moreover, the ionic currents are defined as follows [25].

$$
\begin{aligned}
I_{K}(t) & =g_{K}(t)\left(V_{m}(t)-E_{K}\right), \\
I_{N a}(t) & =g_{N a}(t)\left(V_{m}(t)-E_{N a}\right), \\
I_{L}(t) & =g_{L}(t)\left(V_{m}(t)-E_{L}\right),
\end{aligned}
$$

where $E_{K}, E_{N a}$, and $E_{L}$ are the resting potentials when Potassium, Sodium, and leakage ions are in equilibrium, respectively. Moreover, $g_{K}(t), g_{N a}(t)$, and $g_{L}$ are the conductance of Potassium, Sodium, and leakage ionic channels, respectively. Furthermore, $g_{K}(t)$ and $g_{N a}(t)$ are defined as $g_{K}(t)=\overline{g_{K}} n^{4}(t)$ and $g_{N a}(t)=\overline{g_{N a}} m^{3}(t) h(t)$ in [25], where $\overline{g_{K}}$ and $\overline{g_{N a}}$ are the conductance of Potassium and Sodium ionic channels, respectively, when all the channels are open. $n^{4}(t)$ and $m^{3}(t) h(t)$ give the opening probability of Potassium and Sodium ionic channels, respectively, and can be derived as follows [25].

$$
\begin{aligned}
& \frac{d n}{d t}=\alpha_{n}(t)(1-n)+\beta_{n}(t) n, \\
& \frac{d m}{d t}=\alpha_{m}(t)(1-m)+\beta_{m}(t) m, \\
& \frac{d h}{d t}=\alpha_{h}(t)(1-h)+\beta_{h}(t) h,
\end{aligned}
$$

where $\alpha_{n}(t), \beta_{n}(t), \alpha_{m}(t), \beta_{m}(t), \alpha_{h}(t)$, and $\beta_{h}(t)$ are derived in $\mathrm{ms}^{-1}$ using following equations.

$$
\begin{aligned}
\alpha_{n}(t) & =\frac{0.01\left(10-v_{m}(t)\right)}{\exp \left(1-0.1 v_{m}(t)\right)-1} \\
\beta_{n}(t) & =0.125 \exp \left(\frac{-v_{m}(t)}{80}\right)
\end{aligned}
$$




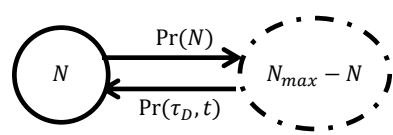

Ready Poo
Unavailable Pool

(a)

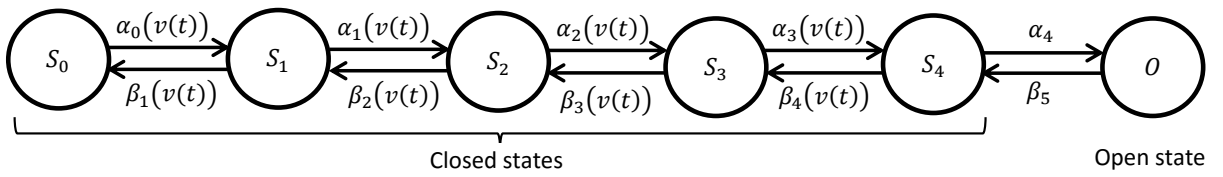

(b)

Fig. 4. Different parts of vesicle release process, (a) Pool-based release from a synaptic terminal, and (b) VDCCs gating.

$$
\begin{aligned}
\beta_{m}(t) & =4 \exp \left(\frac{-v_{m}(t)}{18}\right) \\
\alpha_{m}(t) & =\frac{2.5-0.1 v_{m}(t)}{\exp \left(2.5-0.1 v_{m}(t)\right)-1} \\
\alpha_{h}(t) & =0.07 \exp \left(\frac{-v_{m}(t)}{20}\right) \\
\beta_{h}(t) & =\frac{1}{\exp \left(3-0.1 v_{m}(t)\right)+1}
\end{aligned}
$$

where $v_{m}(t)=V_{m}(t)-V_{\text {rest }}$ in $\mathrm{mV}$ [25].

\section{B. Vesicle Release}

In this section, we describe the vesicle release model having impacts of AP shape variations. In our model, we consider the existence of multiple terminals between a pair of neurons, which enables the release of multiple vesicles to all synapses between an input and output neuron.

1) Release from Single Pre-synaptic Terminal:

a) Pool-based Release Model: To accurately model the vesicle release process, the simple single-pool model depicted in Fig. 4(a) is proposed in [15]. In this figure, $N_{\max }$ is the capacity of RP, i.e., the number of RRVs when the neuron is not stimulated for a long time and all RRVs are recovered, $\operatorname{Pr}(N)$ is the release probability per stimulus when the RP has $N$ vesicles with $N \leq N_{\max }, \tau_{D}$ is the mean recovery time of a vacancy replenishment, and $\operatorname{Pr}\left(\tau_{D}, t\right)$ is the probability of one vacancy replenishment after $t$ second.

To properly model the vesicle release process in hippocampal pyramidal neurons, we consider the univesicular release model, in which at most one vesicle can be released from each terminal. Prior to release of any vesicle, the release of each of RRVs as a result of the spike arrival is independent from others and is governed with a Poisson process with rate $\lambda_{v}(t)$. However, release of one of them transiently prevents fusion of others with membrane [15]. The release or fusion rate for a single vesicle upon arrival of spike is then defined as $\alpha_{v}=\int \lambda_{v}(t) d t$, where the integral is taken over the duration of the spike. Then, the probability of failure in release of a single RRV is $\exp \left(-\alpha_{v}\right)$. When RP contains $N$ vesicles, the failure probability in release can be derived by the $N^{t h}$ power of the failure in release of a single RRV, i.e., $\exp \left(-N \alpha_{v}\right)$. Hence, the probability of having a release, which is the complimentary of failure in release, is $\operatorname{Pr}(N)=1-\exp \left(-N \alpha_{v}\right)$.

In [26], the pool-based model shown in Fig. 4(a) is used to derive the achievable rate of information transmission using neuro-spike communication when the spike shape is constant, i.e., neuron is in control situation. It is shown in [26] that under univesicular release model, the changes in the number of RRVs due to pool-based release model does not affect the performance of communication. Hence, in this paper, we ignore changes in the number of RRVs and consider that replenishment of the released vesicles is done before arrival of next spike to the pre-synaptic terminal, i.e., $N=N_{\max }$.

Since the release is a result of the calcium influx process, $\alpha_{v}$ depends on the concentration of $\mathrm{Ca}^{2+}$ around the RRVs. Depending on the calcium diffusion rate and the structure of pre-synaptic terminal, such as the distance between VDCCs and RRVs and the density of calcium buffers, the concentration of $\mathrm{Ca}^{2+}$ around the RRVs changes from trial to trial [7]. In [27], the average fusion rate in control situation is modeled with $\alpha_{v, C}=0.06 \sqrt{N}$ by using realistic assumptions for concentration of $\mathrm{Ca}^{2+}$ in pre-synaptic neuron. We utilize this model for release rate at control situation and study the relation of $\alpha_{v}$ and $\alpha_{v, C}$ for different spike shapes to find the effect of spike shape variation on vesicle release model. For this aim, we first investigate the impact of spike shape variation on VDCCs' gating. Then, we obtain the relation of calcium influx variation and release process.

b) VDCCs Gating: We can model channel gating of VDCCs by a state diagram as depicted in the Fig. 4(b) [28], where $\left\{S_{0}, S_{1}, S_{2}, S_{3}, S_{4}\right\}$ are closed states, $O$ is the open state and $V_{m}(t)$ is the membrane potential at pre-synaptic terminal. The voltage-dependent forward and backward rates between closed states are calculated by

$$
\begin{aligned}
& \alpha_{i}\left(V_{m}(t)\right)=\alpha_{i} \exp \left(\frac{V_{m}(t)}{V_{i+1}}\right) ; i \in\{0,1,2,3\} \\
& \beta_{i}\left(V_{m}(t)\right)=\beta_{i} \exp \left(\frac{-V_{m}(t)}{V_{i}}\right) ; i \in\{1,2,3,4\}
\end{aligned}
$$

where $\alpha_{i}, \beta_{i}$, and $V_{i}$ are constants related to the type of the VDCC. Then, state equations are

$$
\begin{gathered}
\frac{d S_{0}(t)}{d t}=-\alpha_{0}\left(V_{m}(t)\right) S_{0}(t)+\beta_{1}\left(V_{m}(t)\right) S_{1}(t), \\
\begin{aligned}
\frac{d S_{i}(t)}{d t}= & \alpha_{i-1}\left(V_{m}(t)\right) S_{i-1}(t)-\beta_{i}\left(V_{m}(t)\right) S_{i}(t) \\
& -\alpha_{i}\left(V_{m}(t)\right) S_{i}(t)+\beta_{i+1}\left(V_{m}(t)\right) S_{i+1}(t),
\end{aligned}
\end{gathered}
$$

for $i \in\{1,2,3\}$ and

$$
\begin{aligned}
\frac{d S_{4}(t)}{d t}= & \alpha_{3}\left(V_{m}(t)\right) S_{3}(t)-\beta_{4}\left(V_{m}(t)\right) S_{4}(t) \\
& -\alpha_{4} S_{4}(t)+\beta_{5} O(t), \\
& \frac{d O(t)}{d t}=\alpha_{4} S_{4}(t)-\beta_{5} O(t) .
\end{aligned}
$$

By solving (7)-(10) for each spike shape, we can derive opening probability of a given VDCC. Then, based on [13], [14], the total amount of spike evoked calcium influx through 
each type of VDCCs can be calculated by

$$
C a_{\text {in,type }}^{2+}(t)=\int_{t_{A P}}^{t_{A P}+t} \frac{g_{\text {type }}\left(55 m v-V_{m}(\hat{t})\right) O_{\text {type }}(\hat{t})}{2 e} d \hat{t},
$$

where type $\in\{P / Q, N, R\}, g_{\text {type }}$ and $O_{\text {type }}(t)$ are the conductance and opening probability of each type of VDCCs, respectively, $e$ is the elementary charge, and $C a_{\text {in,type }}^{2+}(t)$ is the total number of calcium ions entering the axonal terminal through single VDCC of the given type $t$ seconds after spike arrival, $t_{A P}$ is the arrival time of AP. By defining $a_{\text {type }}$ as the number of each type of VDCCs in a pre-synaptic terminal, the total number of calcium ions entered the pre-synaptic terminal $t$ seconds after spike arrival is calculated by

$$
C a_{\text {in }}^{2+}(t)=\sum_{\text {type } \in\{P / Q, N, R\}} a_{\text {type }} C a_{\text {in,type }}^{2+}(t) .
$$

c) Effect of Calcium Influx Variation on Release Model: In [29], a Hill equation is used to model the effect of calcium influx changes on EPSP generation in hippocampal pyramidal neurons. The Hill equation providing an empirical representation of the $\mathrm{Ca}^{2+}$ dose-response relationship [30] is

$$
E=S\left(1+\left(\frac{E C_{50}}{C a_{i n}^{2+}(w)}\right)^{N_{H}}\right)^{-1},
$$

where $w$ is the duration of AP, $E$ is the EPSP amplitude, $E C_{50}$ is the concentration of $\mathrm{Ca}^{2+}$ at the half of maximum synaptic response, $S$ is a scaling factor, and $N_{H}$ is the Hill coefficient.

Since only one vesicle can be released per AP from each pre-synaptic terminal in Hippocampal pyramidal neurons and the number of neurotransmitters docked in each vesicle is fix [16], the average change in the EPSP is a result of the variation in the average release probability. On the other hand, the release is governed by Poisson process with mean $\lambda_{v}(t)$, whose integral over the duration of spike gives fusion rate $\alpha_{v}(t)$. Hence, the Hill equation used in [29] can be used to model the relation between changes in neurotransmitter fusion rate and variation in calcium influx as

$$
\frac{\alpha_{v}}{\alpha_{v, C}}=3\left(1+\left(\frac{1.18}{\gamma(w)} \frac{C a_{i n, C}^{2+}}{C a_{i n}^{2+}(w)}\right)^{4.4}\right)^{-1},
$$

where $C a_{i n, C}^{2+}$ and $\alpha_{v, C}$ are the amount of the $C a_{i n}^{2+}(w)$ and $\alpha_{v}$ in the control situation and $\gamma(w)$ models the relation between total calcium influx and concentration of $\mathrm{Ca}^{2+}$ near calcium sensors in pre-synaptic terminal. From our previous work [12], in which the effect of AP width on vesicle release process is modeled, the relation of $\gamma(w)$ and the normalized width can be fitted by

$$
\gamma(w)=\frac{1.62}{\frac{w}{w_{C}}+0.52} .
$$

2) Existence of Multiple Pre-synaptic Terminals: Multiple synapses could exist between a pre- and post-synaptic neuron [31]. The probability of vesicle release from $i^{\text {th }}$ pre-synaptic terminal is $P_{r}\left(N_{i}\right)=1-\exp \left(-N_{i} \alpha_{v, i}\right)$, where $N_{i}=N_{i, \max }$ and $\alpha_{v, i}$ is the fusion rate of this terminal.

Assume that $n_{t}$ synapses exist between input and output neurons and vesicle release from different synapses are independent. Then, the probability of having $k$ release can be calculated with Poisson Binomial distribution with mean $\mu=\sum_{i=1}^{n_{t}} P_{r}\left(N_{i}\right)$ and variance $\sigma^{2}=\sum_{i=1}^{n_{t}} P_{r}\left(N_{i}\right)\left(1-P_{r}\left(N_{i}\right)\right)$ [12]. Thus, the probability of $k$ release is calculated as

$\operatorname{Pr}(k$ release $)=\frac{1}{n_{t}+1} \sum_{l=0}^{n_{t}} D^{-l k} \prod_{m=1}^{n_{t}}\left[1+\left(D^{l}-1\right) \operatorname{Pr}\left(N_{m}\right)\right]$, where $D=\exp \left(\frac{2 i \pi}{n_{t}+1}\right)$ and $i=\sqrt{-1}$ [32]. Then, the probability of vesicle release upon spike arrival can be defined as

$$
\operatorname{Pr}(\text { Release })=\sum_{k=1}^{n_{t}} \operatorname{Pr}(k \text { release }) \text {. }
$$

\section{Diffusion process and Post-Synaptic Neuron Stimulation}

As a result of the randomness in diffusion process, number of available receptors in post-synaptic neuron, and ionic flow, the maximum stimulation of the output neuron in each synaptic terminal varies from trial to trial. In this section, we first consider the response of AMPA and NMDA receptors constant and model the shape of their response. Then, we model the effect of trial to trial variability in the post-synaptic response by multiplying the constant amplitude derived in the first step by a random variable, whose distributions is modeled based on experimental observations [33]. Furthermore, we model the noise in the response of the receptors.

1) EPSP Shaper: Consider $K$ released vesicles and $N_{t}$ bound neurotransmitters per released vesicle. Total number of bound neurotransmitters becomes $j=K N_{N t}$. Since the ratio of AMPA and NMDA receptors current within different synapses to a neuron is constant, we define the number of AMPA receptors, $a$, as a fixed fraction of all bound neurotransmitters, i.e., $a=\lfloor r j\rfloor$, where $r=0.72$ [34] and $\lfloor r j\rfloor$ is the largest integer less than $r j$. The normalized response of both of these receptors to a neurotransmitter is modeled by an alpha function in [2] as $\alpha(t)=\frac{t}{\tau} \exp \left(1-\frac{t}{\tau}\right) u(t)$, where $\tau$ is a constant related to the type of the receptor. Assume $\tau_{1}$ for AMPA and $\tau_{2}$ for NMDA receptors, then, $\tau_{2}>\tau_{1}$.

2) Variable Quantal Amplitude: To model the trial to trial variability in the amplitude of the synaptic response, the response is multiplied by a random variable $q$ with probability distribution of $P(q)$ [35]. According to experimental observations, the amplitude of the post-synaptic response is usually skewed to high amplitudes and can be modeled by a Gamma distribution [33]. Hence, the variable quantal amplitude $q$ is modeled by a gamma distribution in [11] as

$f\left(q ; P_{s}, P_{r}\right)=P_{r}^{P_{s}} \frac{1}{\Gamma\left(P_{s}\right)} q^{P_{s}-1} \exp \left(-P_{r} q\right), q \geq 0, P_{s}, P_{r}>0$,

where $P_{s}=P_{r}=0.6^{-1}$ are shape and rate parameters of gamma distribution, respectively, and $\Gamma\left(P_{s}\right)$ is the gamma function calculated as $\Gamma(\alpha)=\int_{0}^{\infty} x^{\alpha-1} e^{-x} d x$.

3) Noise: Post-synaptic membrane voltage at each receptor is corrupted due to different noise sources like thermal noise and synaptic noise, which is due to multiple accesses to synapse from thousands of other neurons. Since the signal of different neurons has the same random structure and due to 
the central limit theorem, the probability density function of the post-synaptic noise converges to a Gaussian process [2], [35]. In Fig. 3, $W_{i}(t)$ for $1 \leq i \leq j$ is the zero mean Gaussian synaptic noise observed in the response of $i$ th receptor.

\section{Optimum Receiver}

Similar to [2], we use the optimum receiver based on Neyman-Pearson detector for spike generation at post-synaptic neuron as depicted in Fig. 3. Thus, two hypotheses exist for detection, $H_{0}$ when there is no spike in the input, and $H_{1}$ when there is a spike in $S(t)$. These hypotheses are

$$
H_{0}: v=\sum_{i=1}^{j} n_{i} \quad \text { and } \quad H_{1}: v=\sum_{i=1}^{j} q_{i} c_{i}+n_{i}
$$

where $q_{i}$ is the variable quantal amplitude for $i^{t h}$ received signal. For $1 \leq i \leq a, c_{i}=\int_{T_{n}}^{T_{n+1}} \alpha_{A}^{2}(\tau) d \tau=c_{A}$ and $n_{i}=\int_{T_{n}}^{T_{n+1}} W_{i}(\tau) \alpha_{A}(\tau) d \tau$. Moreover, for $a+1 \leq i \leq j, c_{i}=$ $\int_{T_{n}}^{T_{n+1}} \alpha_{N}^{2}\left(\tau-t_{0}\right) d \tau=c_{N}$ and $n_{i}=\int_{T_{n}}^{T_{n+1}} W_{i}(\tau) \alpha_{N}(\tau-$ $\left.t_{0}\right)^{n} d \tau$. Here, $\left[T_{n}, T_{n+1}\right]$ is the range of the desired window, $\alpha_{A}(t)$ and $\alpha_{N}\left(t-t_{0}\right)$ are the response due to binding of a neurotransmitter to AMPA and NMDA receptors, respectively, and $t_{0}>0$ is the delay of NMDA receptors. In [2], the threshold value for decision is derived as

$$
\text { Threshold }=\ln \Omega-\ln \frac{\sigma_{0}}{\sigma_{1}}+\frac{\mu_{1}^{2}}{2 \sigma_{1}^{2}},
$$

where $\Omega$ is the ratio of probability of receiving $v$ when there is a spike and when there is no spike, $\sigma_{0}$ is the variance of $v$ when there is no spike, and $\mu_{1}$ and $\sigma_{1}$ are the mean and variance of $v$ when there is a spike, respectively. These parameters are calculated as follows for our model.

$$
\begin{gathered}
\Omega=\frac{\frac{1}{\sqrt{2 \pi \sigma_{1}^{2}}} \exp \left(-\frac{\left(v-\mu_{1}\right)^{2}}{2 \sigma_{1}^{2}}\right)}{\frac{1}{\sqrt{2 \pi \sigma_{0}^{2}}} \exp \left(-\frac{v^{2}}{2 \sigma_{0}^{2}}\right)} \\
\mu_{1}=E\left[v \mid H_{1}\right]=E\left[\sum_{i=1}^{j} c_{i} E\left[q_{i}\right]\right] \\
\sigma_{0}=\operatorname{Var}\left[v \mid H_{0}\right]=E\left[\sum_{i=1}^{j} \operatorname{Var}\left[n_{i}\right]\right] \\
\sigma_{1}=\operatorname{Var}\left[v \mid H_{1}\right]=E\left[\sum_{i=1}^{j} c_{i}^{2} \operatorname{Var}\left[q_{i}\right]+\operatorname{Var}\left[n_{i}\right]\right]+ \\
\operatorname{Var}\left[\sum_{i=1}^{j} c_{i} E\left[q_{i}\right]\right] .
\end{gathered}
$$

By assuming iid channels and noises, (15)-(17) simplify to

$$
\begin{gathered}
\mu_{1}=\left(f c_{A}+(1-f) c_{N}\right) E[q] E[j] \\
\sigma_{0}=E[j] \operatorname{Var}[n] \\
\sigma_{1}=\left(f c_{A}^{2}+(1-f) c_{N}^{2}\right) E[j] \operatorname{Var}[q]+E[j] \operatorname{Var}[n]+ \\
\left(f c_{A}+(1-f) c_{N}\right)^{2} E[q]^{2} \operatorname{Var}[j],
\end{gathered}
$$

where for $1 \leq i \leq j: E\left[q_{i}\right]=E[q], \operatorname{Var}\left[q_{i}\right]=\operatorname{Var}[q]$, and $\operatorname{Var}\left[n_{i}\right]=\operatorname{Var}[n]$. Mean and variance of $j$ are calculated as

$$
\begin{gathered}
E[j]=N_{N t} E[k]=N_{N t} \sum_{i=1}^{n_{t}} P_{r}\left(N_{i}\right) \\
\operatorname{Var}[j]=N_{N t}^{2} \operatorname{Var}[k]=N_{N t}^{2} \sum_{i=1}^{n_{t}} P_{r}\left(N_{i}\right)\left(1-P_{r}\left(N_{i}\right)\right) .
\end{gathered}
$$

\section{Performance Evaluation}

In this section, we analyze the effect of spike width, $w$, and amplitude, $V_{\text {peak }}$, variation on calcium influx and release rate first. Then, we find the detection error probability of the synaptic communication for different spike shapes. For this aim, we utilize $\mathrm{H}-\mathrm{H}$ model to derive the spike shape in control situation. Then, we use the same shape with different duration and amplitudes to find the impacts of spike shape variations on vesicle release process. Parameters of the synaptic channel model are selected as Table I.

\section{A. Spike Shape in Control Situation}

To derive the spike shape in control situation, we consider stimulation of pre-synaptic neuron with $I_{s}(t)=53 \frac{\mu \mathrm{A}}{\mathrm{cm}^{2}}$ for $1 \mathrm{~ms}$, i.e., $I_{m}(t)=I_{s}(t)$. Moreover, we divide time into tiny time steps, $\Delta \tau=0.1 \mathrm{~ms}$. Thus $\frac{d f(t)}{d t}=g(t)$ can be written as $\frac{\Delta f_{i+1}}{\Delta \tau}=g[i]$ for $\forall f(t), g(t)$, where $\Delta f_{i+1}$ is change in $f(t)$ during $i+1$ th time step and $g[i]$ is the value of $g(t)$ at the end of $i$ th time step, i.e., $g(i \Delta \tau)$. Then, the value of $f(t)$ at the end of $i+1$ th time step is derived as $f[i+1]=f[i]+\Delta f_{i+1}$. Finally, we derive changes in the membrane potential in each time step as a result of this stimulation as follows [25].

1) Initialize $V_{m}[0]=V_{\text {rest }}$ and derive $\alpha_{n}[0], \beta_{n}[0], \alpha_{m}[0]$, $\beta_{m}[0], \alpha_{h}[0]$ using $V_{m}[0]$ in equations given by (4)-(6).

2) Set $i=0$ and derive $n[i]=\left(1+\beta_{n}[i] / \alpha_{n}[i]\right)^{-1}, m[i]=$ $\left(1+\beta_{m}[i] / \alpha_{m}[i]\right)^{-1}$ and $h[i]=\left(1+\beta_{h}[i] / \alpha_{h}[i]\right)^{-1}$.

3) Calculate ionic currents $I_{K}[i], I_{N a}[i]$, and $I_{L}[i]$ using (2).

4) Derive changes in the membrane voltage during $i+1$ th time step based on (1) as

$$
\Delta V_{m, i+1}=\frac{\Delta \tau}{C_{m}}\left(I_{s}[i]-I_{K}[i]-I_{N a}[i]-I_{L}[i]\right) .
$$

5) Calculate $V_{m}[i+1]=V_{m}[i]+\Delta V_{m, i+1}$.

6) Derive $\alpha_{n}[i], \beta_{n}[i], \alpha_{m}[i], \beta_{m}[i], \alpha_{h}[i]$ using $V_{m}[i]$ in equations given by (4)-(6).

7) Find $\Delta n_{i+1}, \Delta m_{i+1}$, and $\Delta h_{i+1}$ using equations given by (3) and calculate $n[i+1], m[i+1]$, and $h[i+1]$.

8) $i=i+1$ and go to step 4 until reaching last time step.

Utilizing the parameters given in Table II, the spike shape in control situation is derived as shown in Fig. 5.

\section{B. Calcium Influx}

To evaluate the impact of spike shape variation on calcium influx process, we first investigate the opening probability of VDCCs, i.e., $O_{P / Q}(t), O_{R}(t)$ and $O_{N}(t)$. These opening probabilities during arrival of spikes with different peak amplitudes and normalized widths are shown in Fig. 6 (a-c) and Fig. 
TABLE I

SIMULATION PARAMETERS FOR SYNAPTIC CHANNEL MODEL

\begin{tabular}{|c|c|c|c|}
\hline Parameter & Symbol & Value & Ref. \\
\hline Probability of spike existence in $S(t)$ & & 0.8 & [2] \\
\hline \multirow{3}{*}{$P / Q$-type VDCCs gating parameters } & $\left\{\alpha_{0}, \alpha_{1}, \alpha_{2}, \alpha_{3}, \alpha_{4}\right\}$ & $\{5.89,9.21,5.2,1823.18,247.71\} \frac{1}{\mathrm{~ms}}$ & \multirow{3}{*}{ [28] } \\
\hline & $\left\{\beta_{1}, \beta_{2}, \beta_{3}, \beta_{4}, \beta_{5}\right\}$ & $\{14.99,6.63,132.8,248.58,8.28\} \frac{1}{\mathrm{~ms}}$ & \\
\hline & $\left\{V_{1}, V_{2}, V_{3}, V_{4}\right\}$ & $\{62.61,33.92,135.08,20.86\} \mathrm{mV}$ & \\
\hline \multirow{3}{*}{$N$-type VDCCs gating parameters } & $\left\{\alpha_{0}, \alpha_{1}, \alpha_{2}, \alpha_{3}, \alpha_{4}\right\}$ & $\{4.29,5.24,4.98,772.63,615.01\} \frac{1}{\mathrm{~ms}}$ & \multirow{3}{*}{ [28] } \\
\hline & $\left\{\beta_{1}, \beta_{2}, \beta_{3}, \beta_{4}, \beta_{5}\right\}$ & $\{5.23,6.63,73.89,692.18,7.68\} \frac{1}{\mathrm{~ms}}$ & \\
\hline & $\left\{V_{1}, V_{2}, V_{3}, V_{4}\right\}$ & $\{68.75,39.53,281.62,18.46\} \mathrm{mV}$ & \\
\hline \multirow{3}{*}{ R-type VDCCs gating parameters } & $\left\{\alpha_{0}, \alpha_{1}, \alpha_{2}, \alpha_{3}, \alpha_{4}\right\}$ & $\{9911.36,4.88,4,256.41,228.83\} \frac{1}{\mathrm{~ms}}$ & \multirow{3}{*}{ [28] } \\
\hline & $\left\{\beta_{1}, \beta_{2}, \beta_{3}, \beta_{4}, \beta_{5}\right\}$ & $\{0.62,21.19,51.3,116.97,1.78\} \frac{1}{\mathrm{~ms}}$ & \\
\hline & $\left\{V_{1}, V_{2}, V_{3}, V_{4}\right\}$ & $\{67.75,50.94,173.29,16.92\} \mathrm{mV}$ & \\
\hline Conductance of VDCCs & $g_{P / Q}=g_{N}=g_{R}$ & $2.7 \mathrm{pS}$ & {$[14]$} \\
\hline Mean number of $P / Q-, N-$, and $R$-type VDCCs & $a_{P / Q}, a_{N}$, and $a_{R}$ & 15,16, and 1.5 & {$[14]$} \\
\hline Mean and Variance of quantal amplitude per receptor & $E[q]$ and $\operatorname{Var}[q]$ & $\frac{1}{N_{N t}}$ and $\left(\frac{0.6}{N_{N t}}\right)^{2}$ & [11] \\
\hline Capacity of RP in $i^{\text {th }}$ terminal & $N_{\max , i}$ & 11 & [36] \\
\hline Number of bound neurotransmitters per vesicle & $N_{N t}$ & 11 & [34] \\
\hline Peak and time to peak for EPSP waveform of AMPAs & $h_{\max , A}$ and $\tau_{1}$ & $1 \mathrm{mV}$ and $8 \mathrm{~ms}$ & {$[2]$} \\
\hline Peak and time to peak for EPSP waveform of NMDAs & $h_{\max , N}$ and $\tau_{2}$ & $1 \mathrm{mV}$ and $10 \mathrm{~ms}$ & {$[2]$} \\
\hline Start time of $n^{\text {th }}$ and $(n+1)^{\text {th }}$ window & $T_{n}$ and $T_{n+1}$ & 0 and $150 \mathrm{~ms}$ & \\
\hline Mean and variance of the noise in detection process & $E[n]$ and $\operatorname{Var}[n]$ & 0 and 0.01 & {$[11]$} \\
\hline
\end{tabular}

TABLE II

PARAMETERS OF H-H MODEL [25]

\begin{tabular}{|l|l|l|}
\hline Parameter & Symbol & Value \\
\hline \hline Membrane resting potential & $V_{\text {rest }}$ & $-60 \mathrm{mV}$ \\
\hline Membrane capacitance & $C_{m}$ & $4 \frac{\mu \mathrm{F}}{\mathrm{cm}^{2}}$ \\
\hline \multirow{3}{*}{ Parameters of ionic currents } & $\overline{g_{K}}, E_{K}$ & $0.3 \frac{\mathrm{mS}}{\mathrm{cm}^{2}},-72 \mathrm{mV}$ \\
\cline { 2 - 3 } & $\overline{g_{N a}}, E_{N a}$ & $36 \frac{\mathrm{mS}}{\mathrm{cm}^{2}}, 55 \mathrm{mV}$ \\
\cline { 2 - 3 } & $g_{L}, E_{L}$ & $120 \frac{\mathrm{mS}}{\mathrm{cm}^{2}},-49.38 \mathrm{mV}$ \\
\hline
\end{tabular}

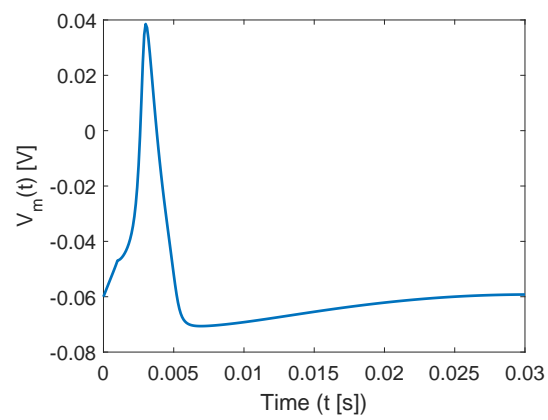

Fig. 5. Spike shape in control situation derived by utilizing H-H model.

6 (d-f), respectively. As illustrated in Fig. 6, both amplitude, i.e., $V_{\text {peak }}$, and width, i.e., $w$, of the spike affect the opening probability of VDCCs. Moreover, spike width variations have impact on both width and peak amplitude of opening probabilities. However, changes in the spike amplitude only affect the maximum opening probabilities. The reason is that (i) by increasing the width of spike the VDCCs receive voltage for a longer time, which improves their opening probability and at the same time keeps them open for longer time and (ii) higher spike peak amplitudes improve the opening probability of VDCCs but do not affect their opening time.

Total number of calcium ions entering the pre-synaptic terminal $t$ seconds after arrival of a spike at time $t_{A P}$ is derived by substituting (11) in (12). Hence, considering a fixed shape of spike, the important parameters controlling the total number of Calcium ions entering the pre-synaptic terminal are (i) conductance of different channels, i.e., $g_{\text {type }}$, (ii) the number of available channels from each type, $a_{\text {type }}$, and (iii) the opening probability of each channel, $O_{\text {type }}(\hat{t})$ for $t_{A P}<\hat{t}<t_{A P}+t$ and type $\in\{P / Q, R, N\}$. Since $g_{P / Q}=g_{N}=g_{R}$ and $a_{R}$ is far less than $a_{P / Q}$ and $a_{N}$ [14], the $R$-type VDCCs have the lowest impact on the total number of Calcium ions entering the pre-synaptic terminal and the vesicle release process downstream of it. Moreover, as shown in Fig. 6, range of changes in $O_{P / Q}(t)$ is less than range of changes in $O_{N}(t)$ as a result of both spike width and amplitude variation. Hence, $N$-type VDCCs have the highest effect on the changes in total Calcium ions entering the presynaptic terminal as a result of spike shape variation.

Variations in the opening probability of VDCCs have direct impact on the calcium influx to the pre-synaptic terminal upon arrival of the spike. Hence, we illustrate the total number of calcium ions entering the pre-synaptic terminal as a result of arrival of spikes with different widths and amplitudes, i.e., $\mathrm{Ca}_{i n}^{2+}(w)$, normalized to its value in control situation, i.e., $C a_{i n, C}^{2+}$, in Fig. 7. As depicted in Fig. 7, increasing width of the spike intensifies the total number of calcium ions entering the pre-synaptic terminal, i.e., $C a_{i n}^{2+}(w)$. Moreover, increasing spike amplitude first improves the calcium influx and then worsens it. The reason of this trend can be found by analyzing the equation used for deriving $C a_{i n}^{2+}(w)$. According to (11), changes in both $55 \mathrm{mv}-V_{m}(t)$ and $O_{\text {type }}(t)$ as a result of 


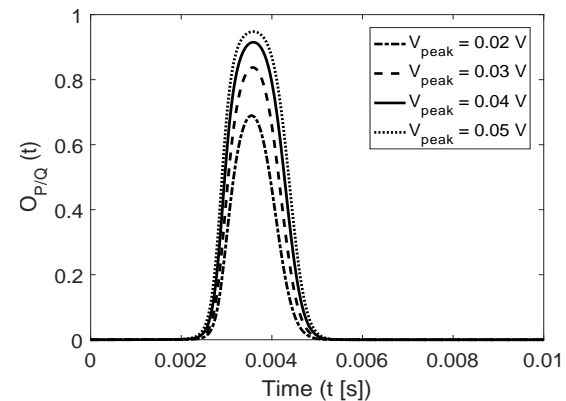

(a) Normalized width $=1$

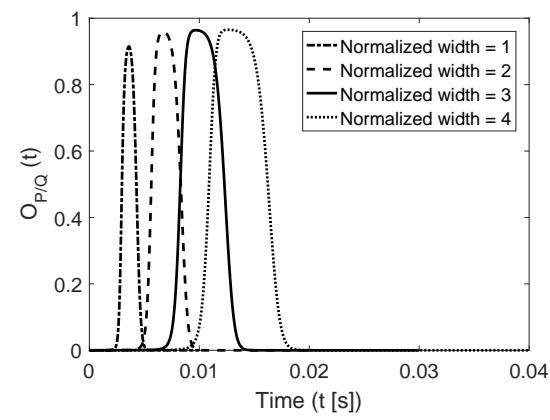

(d) $V_{\text {peak }}=0.04 \mathrm{~V}$

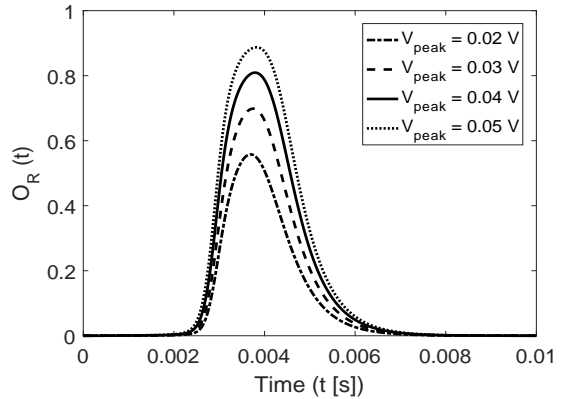

(b) Normalized width $=1$

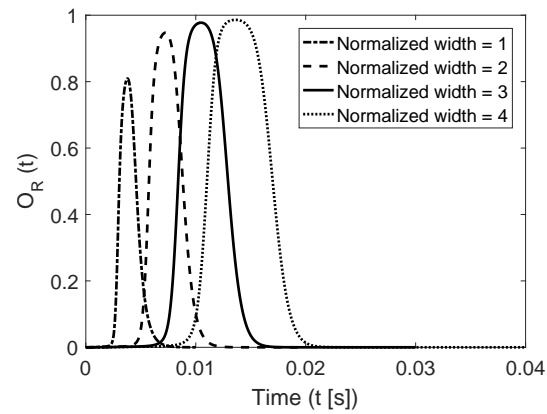

(e) $V_{\text {peak }}=0.04 \mathrm{~V}$

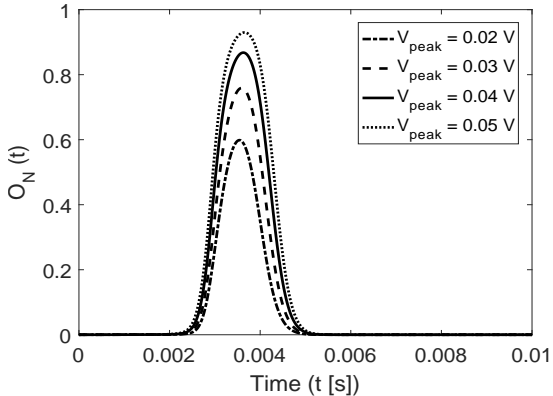

(c) Normalized width $=1$

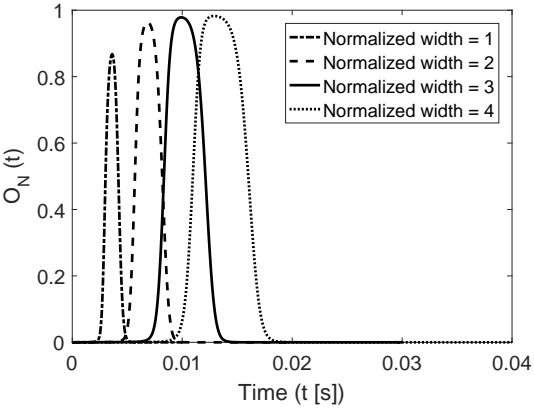

(f) $V_{\text {peak }}=0.04 \mathrm{~V}$

Fig. 6. Opening probability of P/Q-, R-, and N-type VDCCs upon arrival of spikes with different (a-c) amplitudes, $V_{p e a k}$, and (d-f) widths, $w$.

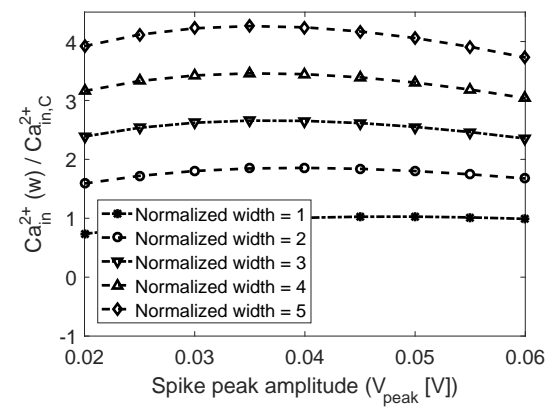

Fig. 7. Changes in the total number of calcium ions entering the pre-synaptic terminal as a result of arrival of spikes with different widths and amplitudes, i.e., $C a_{i n}^{2+}(w)$, normalized to its value in control situation, i.e., $C a_{i n, C}^{2+}$.

increasing $V_{\text {peak }}$ are important in calculating $C a_{i n}^{2+}(w)$. While increasing $V_{\text {peak }}$ increases $O_{\text {type }}(t)$, it decreases $55 \mathrm{mv}-$ $V_{m}(t)$. Moreover, as it can be seen in Fig. 6 (a-c), increment in $O_{\text {type }}(t)$ is decreasing by increasing $V_{\text {peak }}$. Hence, after some point, the reduction in $55 \mathrm{mv}-V_{m}(t)$ become dominant compared to increment of $O_{\text {type }}(t)$, thus, $C a_{i n}^{2+}(w)$ decreases by increasing $V_{\text {peak }}$ after that point.

As illustrated in Fig. 7, variations in the width of spike affects $\mathrm{Ca}_{i n}^{2+}(w)$ more than changes in the spike amplitude. The timing of calcium influx upon spike arrival at $t_{A P}=0$ is depicted in Fig. 8. As shown in Fig. 8 changes in the spike amplitude does not change the duration of calcium influx, however, for higher spike widths, the calcium ions enter to the pre-synaptic terminal for a longer time. The reason of this difference can be found in Fig. 6 as the VDCCs stay open for a longer time by increasing width of spike.

\section{Vesicle Release}

The effect of spike width and amplitude variation on the release rate is plotted in Fig. 9(a). Based on this figure, the spike width has a direct relation with release rate as it directly influences the amount of calcium ions entering the neuron. Furthermore, the improvement in release rate decreases by increasing spike width, which is in contrast with the observations in Fig. 7, where the rate of change in $\mathrm{Ca}_{\text {in }}^{2+}(w)$ as a result of increasing spike width does not depend on the spike width. This is due to using (13) to calculate release rate, where $\gamma(w)$ decreases by increasing spike width according to (14). Moreover, increasing spike peak amplitude, $V_{\text {peak }}$, first improves the release rate and then worsens it since same pattern was observed for impact of changes in $V_{\text {peak }}$ on $\mathrm{Ca}_{\text {in }}^{2+}(w)$. In addition, increasing $V_{\text {peak }}$ decreases the impact of spike width variations on the release rate. The vesicle release probability, $\operatorname{Pr}(N)$, after arrival of spikes with different widths and peak amplitudes is plotted in Fig. 9(b). As illustrated in this figure, the increment in spike shape and peak amplitude does not contribute to change vesicle release probability as independent factors. While $\operatorname{Pr}(N)$ is very sensitive to spike amplitude variations when the spike has its control width, this sensitivity reduces radically by increasing the spike width. Furthermore, increasing the spike peak amplitude decreases the enhancement of release probability as a result of increasing spike width.

As shown in Fig. 9(b), doubling the spike width from its control value improves the vesicle release probability as a result of increment in release rate observed in Fig. 9(a). However, further increase in spike width does not have significant impact on vesicle release probability, $\operatorname{Pr}(N)$. Moreover, Fig. 7 and Fig. 9 depict that the increase of calcium influx due to 


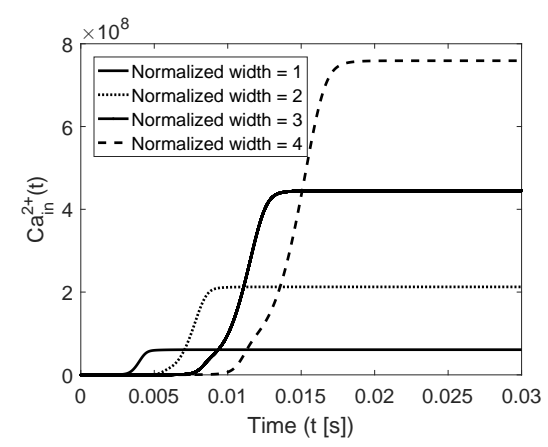

(a) $V_{\text {peak }}=0.04 \mathrm{~V}$, different spike widths

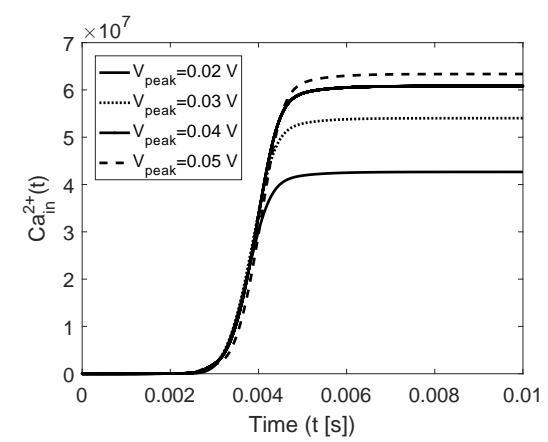

(b) Normalized width $=1$, different spike amplitudes

Fig. 8. Total calcium ions entering pre-synaptic terminal, i.e., $C a_{i n}^{2+}(t)$, as a function of time upon arrival of spikes with different shapes.

changes in spike amplitude affects the vesicle release process more than when the reason is spike width variation.

Next, we plot the expected number of released vesicles to all synapses between two neurons as a result of arrival of spikes with different shape parameter in Fig. 10. As shown in Fig. 10 (a-c) wider spikes lead to release of more number of vesicles to all synapses between two neurons. The reason is the improvement of vesicle release probability by increasing spike width as shown in Fig. 9(b). Moreover, increasing spike peak amplitude reduces the impact of spike width variations on number of released vesicles since it affects the vesicle release probability in the same manner as shown Fig. 9(b).

As depicted in Fig. 10 (d-f), increasing $V_{\text {peak }}$ while spike width is fix first causes release of more and then less vesicles to all synapses among two neurons since the same pattern is observed for vesicle release probability in Fig. 9(b). Moreover, increasing the spike width decreases the impact of spike amplitude variation on vesicle release process.

As illustrated in Fig. 10, the expected number of released vesicles to all synapses between two neurons cannot be greater than number of synapses since at most one vesicle can be released per synapse in hippocampal pyramidal neurons. Moreover, increasing number of synapses between two neurons increases the expected number of released vesicles per spike, which, in turn, enhances the number of bound neurotransmitters with post-synaptic receptors.

\section{Error Probability}

In this section, we evaluate the impact of spike shape variation on the probability of having error in detecting the

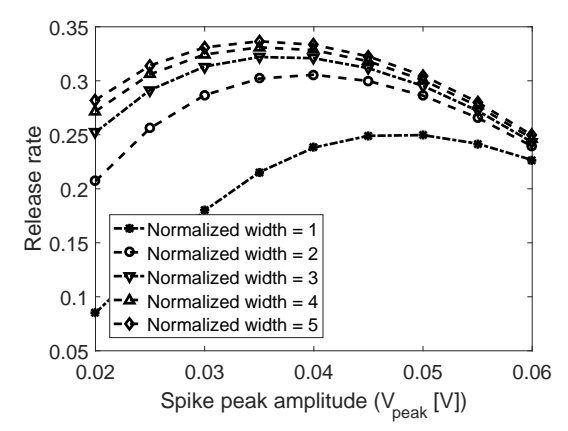

(a)

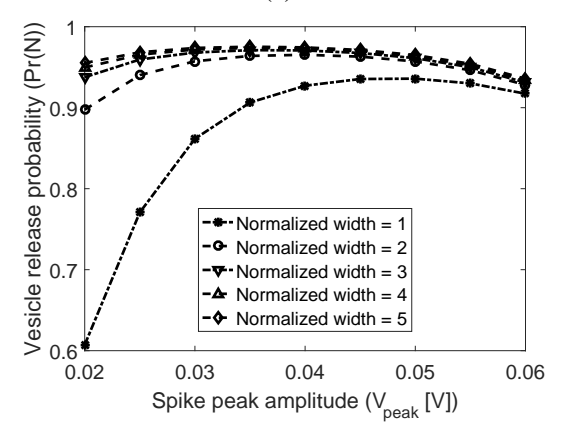

(b)

Fig. 9. Changes in (a) release rate and (b) vesicle release probability as a result of spike width and amplitude variation.

transmitted signal over the communication model shown in Fig. 3. As depicted in Fig. 11 (a-c), increasing spike width decreases the error detection probability, which is a direct result of improving the vesicle release probability. Moreover, increasing spike amplitude decreases impact of spike width variation on the error probability as similar trend was observed for vesicle release probability.

As illustrated in Fig. 11 (d-f), increasing spike peak amplitude, $V_{\text {peak }}$, first reduces the error detection probability, i.e., improves the performance of the synaptic communication channel by increasing the probability of correct detection of the transmitted signal, and then increases this error. The reason is the observation of the same pattern for vesicle release probability in Fig. 9(b). Moreover, increasing the spike width decreases the impact of spike amplitude variation on the probability of error detection. Based on Fig. 11, increasing number of terminals between a pair of neuron, i.e., $n_{t}$, decreases the error probability. The reason is that increasing $n_{t}$ enhances the probability of having more bound neurotransmitters to the post-synaptic terminal, which increases the chance of correctly detecting the input when there is a spike at input.

Note that increasing spike width decreases the re-usability of communication channel since longer time is used for transmitting each spike. Moreover, refractory period of the neuron, i.e., the duration that neuron is not able to fire another AP after firing one, is also an important factor depicting the re-usability of the neuro-spike communication channel. The broadening of spike width can happen in two ways, (i) as a result of slow recovery of $K^{+}$channels from inactivation state if the neuron receives high frequency stimulation [5] or (ii) by manipulating the ionic channels existing in the axon [37]. In the first case, 


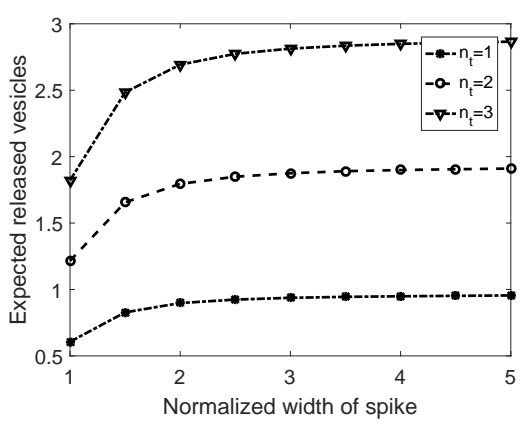

(a) $V_{\text {peak }}=0.02 \mathrm{~V}$

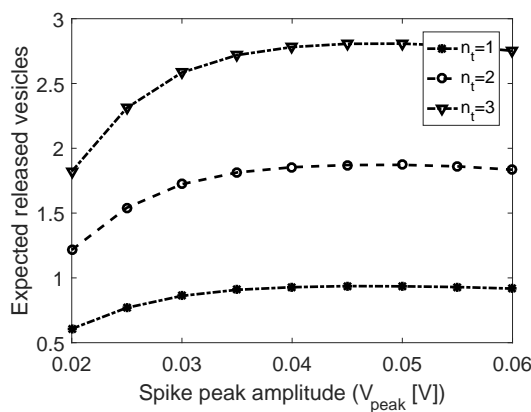

(d) Normalized width $=1$

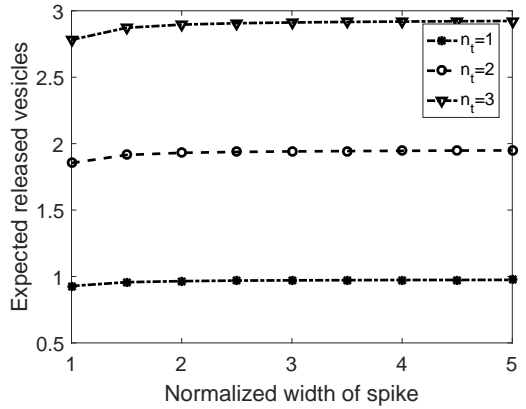

(b) $V_{\text {peak }}=0.04 \mathrm{~V}$

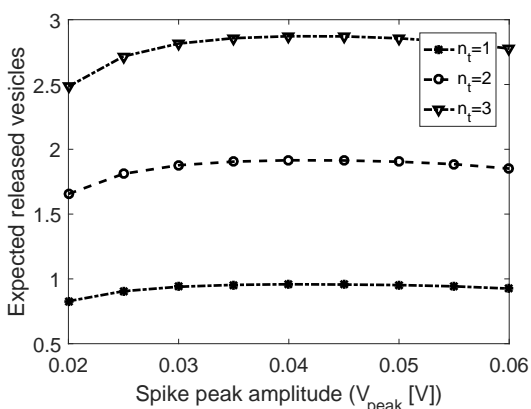

(e) Normalized width $=2$

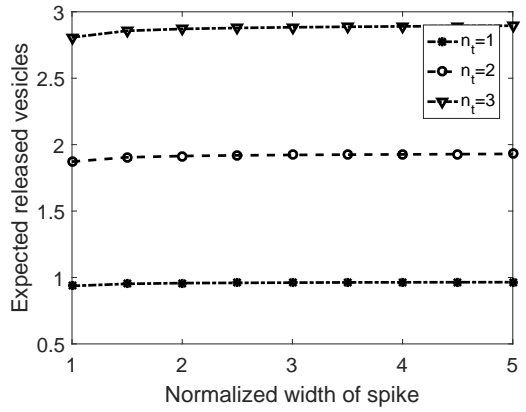

(c) $V_{\text {peak }}=0.05 \mathrm{~V}$

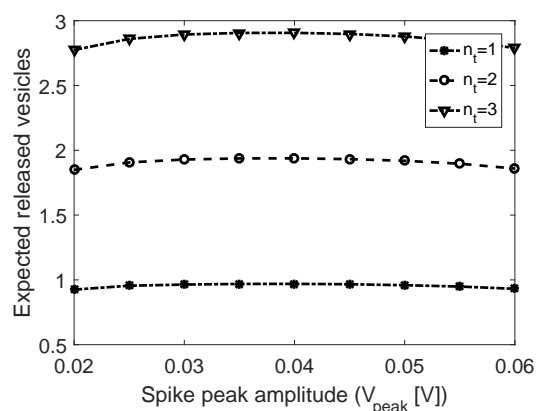

(f) Normalized width $=4$

Fig. 10. Expected number of released vesicles to all synapses between two neurons for different (a-c) spike widths and (d-f) spike amplitudes.

the refractory period of the neuron also changes depending on the previous activities of the axon [6]. However, depending on the technique used for manipulation of ionic channels, more experimental studies are required to find possible changes in refractory period in the second case. Furthermore, increasing the spike amplitude is very costly for nanomachines since it requires more transmission power. Hence, two trade offs exists in designing nanonetworks utilizing neuro-spike communication paradigm, (i) one between the channel re-usability and detection error, and (ii) one between the improvement achieved in detection error and increment in power consumption caused by increasing spike amplitude. The error probability of the channel by neglecting outcomes of spike shape variations is the same as plotted error probability with normalized width equal to 1 and $V_{\text {peak }}=40 \mathrm{mV}$. Thus, we see that the changes in both of spike width and amplitude affect the efficiency of the synaptic channel, which will affect the effectiveness of the neuro-spike communication. Moreover, we have observed that changes in the spike width and amplitude do not affect the performance of the system as two independent factors. Hence, a proper model for neuro-spike communication should contain effects of changes in spike shape during axonal transmission on both synaptic propagation and spike generation mechanisms. This will enable us to accurately explain the performance of either an artificial communication system working based on neuro-spike communication paradigm or estimate the functionality of neurons being stimulated by biological or artificial nanomachines. Moreover, the simulation results illustrated in this section give insight in selecting spike shape parameters to reach the optimal performance in a nanonetwork designed based on neuro-spike communication paradigm.

\section{CONCLUSION}

While the shape of AP is independent from the strength of the applied stimulus, it can change during axonal propagation depending on the activation states of ionic channels. In this paper, we proposed a complete synaptic channel model that captures impacts of AP shape variations. To this aim, we proposed a single-pool based model for release of vesicles from pre-synaptic terminal. Then, we used a six-states state diagram to model the kinetics of VDCCs and found the impact of calcium influx variation on vesicle release process. Finally, we derived the structure of optimum detector for our synaptic communication model to find the consequences of spike shape variation in functionality of synaptic transmission. Simulation results depicted that both changes in spike amplitude and width influence the efficiency of synaptic communication, which in turn affects the effectiveness of neuro-spike communication.

\section{REFERENCES}

[1] O. B. Akan et al., "Fundamentals of molecular information and communication science," Proc. IEEE, vol. 105, no. 2, pp. 306-318, 2017.

[2] E. Balevi and O. B. Akan, "A physical channel model for nanoscale neuro-spike communications," IEEE Trans. Commun., vol. 61, no. 3, pp. 1178-1187, 2013.

[3] M. F. Bear et al., Neuroscience: Exploring the Brain, 3rd ed., 2007.

[4] H. Ramezani and O. B. Akan, "A communication theoretical modeling of axonal propagation in hippocampal pyramidal neurons," IEEE Trans. Nanobiosci., 2017.

[5] J. R. Geiger and P. Jonas, "Dynamic control of presynaptic ca 2+ inflow by fast-inactivating $\mathrm{k}+$ channels in hippocampal mossy fiber boutons," Neuron, vol. 28, no. 3, pp. 927-939, 2000.

[6] Z. Feng et al., "High frequency stimulation extends the refractory period and generates axonal block in the rat hippocampus," Brain stimulation, vol. 7, no. 5, pp. 680-689, 2014.

[7] H. Ramezani and O. B. Akan, "Importance of vesicle release stochasticity in neuro-spike communication," in 39th IEEE EMBC Conf. IEEE, 2017, pp. 3343-3347. 


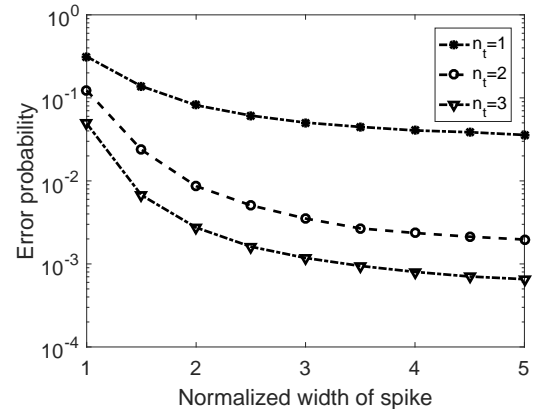

(a) $V_{\text {peak }}=0.02 \mathrm{~V}$

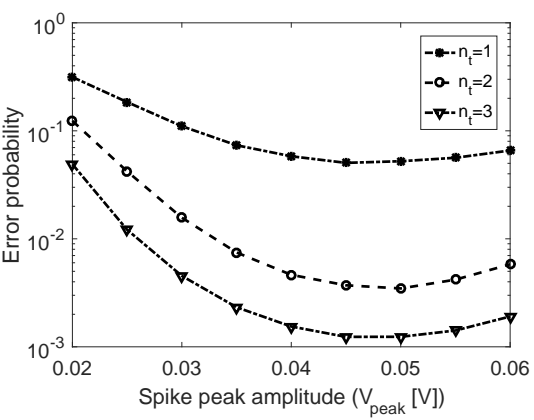

(d) Normalized width $=1$

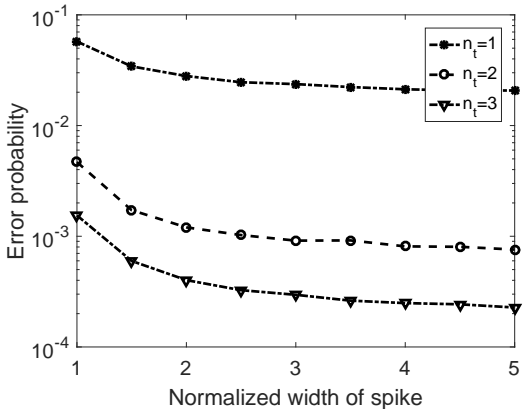

(b) $V_{\text {peak }}=0.04 \mathrm{~V}$

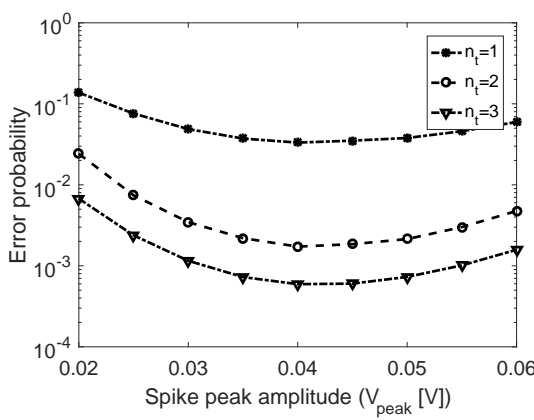

(e) Normalized width $=2$

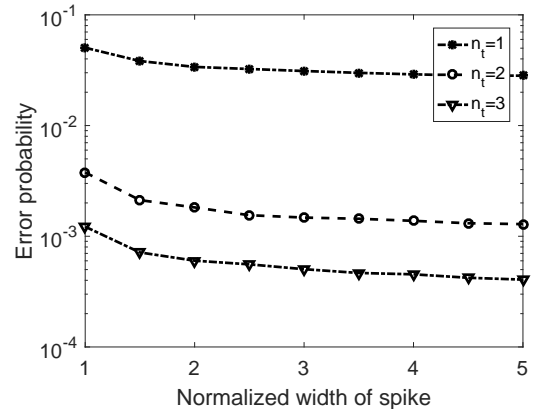

(c) $V_{\text {peak }}=0.05 \mathrm{~V}$

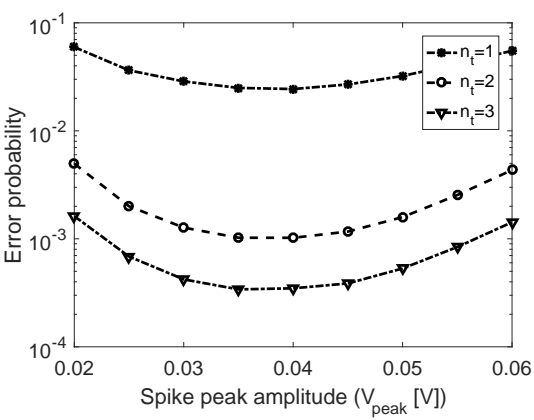

(f) Normalized width $=4$

Fig. 11. Error probability for different number of synaptic terminals under different (a-c) spike widths and (d-f) spike amplitudes.

[8] L. Galluccio et al., "Modeling signal propagation in nanomachine-toneuron communications," Nano Commun. Netw., vol. 2, no. 4, pp. 213222, 2011

[9] —- "Characterization of molecular communications among implantable biomedical neuro-inspired nanodevices," Nano Commun. Netw., vol. 4, no. 2, pp. 53-64, 2013.

[10] M. Veletić et al., "From nano-scale neural excitability to long term synaptic modification," in Proceedings of 1st ACM NanoCom Conf. ACM, 2014, p. 22.

[11] D. Malak and O. B. Akan, "A communication theoretical analysis of synaptic multiple-access channel in hippocampal-cortical neurons," IEEE Trans. Commun., vol. 61, no. 6, pp. 2457-2467, 2013.

[12] H. Ramezani and O. B. Akan, "Synaptic channel model including effects of spike width variation," in Proc. ACM NANOCOM. ACM, 2015, p. 11.

[13] M. B. Hoppa et al., "Control and plasticity of the presynaptic action potential waveform at small cns nerve terminals," Neuron, vol. 84, no. 4 pp. 778-789, 2014.

[14] Y. S. Ermolyuk et al., "Differential triggering of spontaneous glutamate release by p/q-, n-and r-type ca2+ channels," Nature Neurosci., vol. 16, no. 12 , pp. 1754-1763, 2013.

[15] V. Matveev and X.-J. Wang, "Implications of all-or-none synaptic transmission and short-term depression beyond vesicle depletion: a computational study," J. Neurosci., vol. 20, no. 4, pp. 1575-1588, 2000.

[16] D. Sulzer and R. Edwards, "Vesicles: equal in neurotransmitter concentration but not in volume," Neuron, vol. 28, no. 1, pp. 1-9, 2000.

[17] A. Alabi and R. W. Tsien, "Synaptic vesicle pools and dynamics," Cold Spring Harbor perspectives in biol., vol. 4, no. 8, p. a013680, 2012.

[18] C. Heinemann et al., "A two-step model of secretion control in neuroendocrine cells," Pflügers Archiv, vol. 424, no. 2, pp. 105-112, 1993.

[19] S. O. Rizzoli and W. J. Betz, "Synaptic vesicle pools," Nature Rev. Neurosci., vol. 6, no. 1, pp. 57-69, 2005.

[20] P. Andersen et al., The Hippocampus Book, 2006.

[21] A. J. Watt et al., "Activity coregulates quantal ampa and nmda currents at neocortical synapses," Neuron, vol. 26, no. 3, pp. 659-670, 2000.

[22] J. C. Magee and E. P. Cook, "Somatic epsp amplitude is independent of synapse location in hippocampal pyramidal neurons," Nature Neurosci., vol. 3, no. 9, pp. 895-903, 2000 .

[23] D. A. Nicholson et al., "Distance-dependent differences in synapse number and ampa receptor expression in hippocampal cal pyramidal neurons," Neuron, vol. 50, no. 3, pp. 431-442, 2006.

[24] M. A. Smith et al., "Mechanism of the distance-dependent scaling of schaffer collateral synapses in rat ca1 pyramidal neurons," J. physiology, vol. 548, no. 1, pp. 245-258, 2003.

[25] R. Plonsey and R. C. Barr, Bioelectricity: a quantitative approach. Springer Science \& Business Media, 2007.

[26] H. Ramezani et al., "Rate region analysis of multi-terminal neuronal nanoscale molecular communication channel," in Proc. IEEE Nano. IEEE, 2017.

[27] L. E. Dobrunz and C. F. Stevens, "Heterogeneity of release probability, facilitation, and depletion at central synapses," Neuron, vol. 18, no. 6 , pp. 995-1008, 1997.

[28] L. Li et al., "Differential gating and recruitment of p/q-, n-, and $\mathrm{r}$ type ca2+ channels in hippocampal mossy fiber boutons," J. Neurosci., vol. 27, no. 49, pp. 13420-13 429, 2007.

[29] J. Qian and P. Saggau, "Modulation of transmitter release by action potential duration at the hippocampal ca3-ca1 synapse," J. neurophysiology, vol. 81, no. 1, pp. 288-298, 1999.

[30] C. A. Reid et al., "N-and p/q-type ca2+ channels mediate transmitter release with a similar cooperativity at rat hippocampal autapses," $J$. Neurosci., vol. 18, no. 8, pp. 2849-2855, 1998.

[31] G. M. Shepherd and K. M. Harris, "Three-dimensional structure and composition of ca3 ca1 axons in rat hippocampal slices: implications for presynaptic connectivity and compartmentalization," J. Neurosci., vol. 18 , no. 20 , pp. $8300-8310,1998$.

[32] M. Fernandez and S. Williams, "Closed-form expression for the poissonbinomial probability density function," IEEE Trans. Aerosp. Electron. Syst., vol. 46, no. 2, pp. 803-817, 2010.

[33] J. Bekkers et al., "Origin of variability in quantal size in cultured hippocampal neurons and hippocampal slices." Proceedings of the National Academy of Sciences, vol. 87, no. 14, pp. 5359-5362, 1990.

[34] E. A. Nimchinsky et al., "The number of glutamate receptors opened by synaptic stimulation in single hippocampal spines," J. Neurosci., vol. 24 no. 8, pp. 2054-2064, 2004.

[35] A. Manwani and C. Koch, "Detecting and estimating signals over noisy and unreliable synapses: information-theoretic analysis," Neural computation, vol. 13, no. 1, pp. 1-33, 2001.

[36] T. Schikorski and C. F. Stevens, "Quantitative ultrastructural analysis of hippocampal excitatory synapses," J. Neurosci., vol. 17, no. 15, pp. 5858-5867, 1997.

[37] B. L. Sabatini and W. G. Regehr, "Control of neurotransmitter release by presynaptic waveform at the granule cell to purkinje cell synapse," Journal of Neuroscience, vol. 17, no. 10, pp. 3425-3435, 1997. 